\title{
PERTURBATION BOUNDS OF P-MATRIX LINEAR COMPLEMENTARITY PROBLEMS *
}

\author{
XIAOJUN $\mathrm{CHEN}^{\dagger}$ AND SHUHUANG XIANG ${ }^{\ddagger}$
}

\begin{abstract}
We define a new fundamental constant associated with a P-matrix and show that this constant has various useful properties for the P-matrix linear complementarity problems (LCP). In particular, this constant is sharper than the Mathias-Pang constant in deriving perturbation bounds for the P-matrix LCP. Moreover, this new constant defines a measure of sensitivity of the solution of the P-matrix LCP. We examine how perturbations in the data affect the solution of the LCP and efficiency of Newton-type methods for solving the LCP.
\end{abstract}

Key words. Perturbation bounds, sensitivity, linear complementarity problems

AMS subject classifications. 90C $33,65 \mathrm{G} 20,65 \mathrm{G} 50$

1. Introduction. The linear complementarity problem is to find a vector $x \in R^{n}$ such that

$$
M x+q \geq 0, \quad x \geq 0, \quad x^{T}(M x+q)=0,
$$

or to show that no such vector exists, where $M \in R^{n \times n}$ and $q \in R^{n}$. We denote this problem by $\operatorname{LCP}(M, q)$. A matrix $M$ is called a P-matrix if its all principal minors are positive, which is equivalent to

$$
\max _{1 \leq i \leq n} x_{i}(M x)_{i}>0 \text { for all } x \neq 0 .
$$

It is well-known that $M$ is a $\mathrm{P}$-matrix if and only if the $\operatorname{LCP}(M, q)$ has a unique solution for any $q \in R^{n}$ [3]. Moreover, if $M$ is a P-matrix, then there is a neighborhood $\mathcal{M}$ of $M$, such that all matrices in $\mathcal{M}$ are $\mathrm{P}$-matrices. Hence, we can define a solution function $x(A, b): \mathcal{M} \times R^{n} \rightarrow R_{+}^{n}$, where $x(A, b)$ is the solution of $\operatorname{LCP}(A, b)$ and $R_{+}^{n}=\left\{x \in R^{n} \mid x \geq 0\right\}$.

In [12], Mathias and Pang introduced the following fundamental quantity associated with a P-matrix,

$$
c(M)=\min _{\|x\|_{\infty}=1} \max _{1 \leq i \leq n}\left\{x_{i}(M x)_{i}\right\} .
$$

This constant has often been used in error analysis of the LCP [2,3]. In particular, the following Lemma has been widely applied in perturbation bounds.

Lemma 1.1. [3] Let $M \in R^{n \times n}$ be a P-matrix. The following statements hold:

(i) for any two vectors $q$ and $p$ in $R^{n}$,

$$
\|x(M, q)-x(M, p)\|_{\infty} \leq \frac{1}{c(M)}\|q-p\|_{\infty}
$$

(ii) for each vector $q \in R^{n}$, there exist a neighborhood $\mathcal{U}$ of the pair $(M, q)$ and a constant $c_{0}>0$ such that for any $(A, b),(B, p) \in \mathcal{U}, A, B$ are $P$-matrices and

$$
\|x(A, b)-x(B, p)\|_{\infty} \leq c_{0}\left(\|A-B\|_{\infty}+\|b-p\|_{\infty}\right) .
$$

\footnotetext{
* This work is partly supported by a Grant-in-Aid from Japan Society for the Promotion of Science.

${ }^{\dagger}$ Department of Mathematical Sciences, Hirosaki University, Hirosaki 036-8561, Japan, chen@cc.hirosaki-u.ac.jp.

${ }_{\ddagger}$ Department of Applied Mathematics and Software, Central South University, Changsha, Hunan 410083, China, xiangsh@mail.csu.edu.cn
} 
Lemma 1.1 shows that when $M$ is a $\mathrm{P}$-matrix, for each $q, x(A, b)$ is a locally Lipschitzian function of $(A, b)$ in a neighborhood of $(M, q)$, and $x(M, b)$ is a globally Lipschitzian function of $b$. This property plays a very important role in the study of the LCP and mathematical programs with LCP constraints [11]. However, the constant $c(M)$ is difficult to compute, and $c_{0}$ is not specified. It is hard to use this lemma for verifying accuracy of a computed solution of the LCP when the data $(M, q)$ contain errors.

In this paper, we introduce a new constant for a P-matrix,

$$
\beta_{p}(M)=\max _{d \in[0,1]^{n}}\left\|(I-D+D M)^{-1} D\right\|_{p}
$$

where $D=\operatorname{diag}(d)$ with $0 \leq d_{i} \leq 1, i=1,2, \ldots, n$ and $\|\cdot\|_{p}$ is the matrix norm induced by the vector norm for $p \geq 1$.

Using the constant $\beta_{p}(M)$, we give perturbation bounds for $M$ being a P-matrix as follows.

$$
\begin{gathered}
\|x(M, q)-x(M, p)\|_{p} \leq \beta_{p}(M)\|q-p\|_{p} \\
\|x(A, b)-x(B, p)\|_{p} \leq \frac{\beta_{p}(M)^{2}}{(1-\eta)^{2}}\left\|(-p)_{+}\right\|\left\|_{p}\right\| A-B\left\|_{p}+\frac{\beta_{p}(M)}{1-\eta}\right\| b-p \|_{p},
\end{gathered}
$$

and

$$
\frac{\|x(M, q)-x(A, b)\|_{p}}{\|x(M, q)\|_{p}} \leq \frac{2 \epsilon}{1-\eta} \beta_{p}(M)\|M\|_{p},
$$

where $\eta \in[0,1)$ and $\epsilon>0$ can be chosen, $A, B \in \mathcal{M}:=\left\{A \mid \beta_{p}(M)\|M-A\|_{p} \leq \eta\right\}$, and $\|q-b\|_{p} \leq \epsilon\left\|(-q)_{+}\right\|_{p}$.

The constant $\beta_{p}(M)$ has the following interesting properties.

- If $M$ is a P-matrix, then for $\|\cdot\|_{\infty}$,

$$
\beta_{\infty}(M) \leq \frac{1}{c(M)}
$$

- If $M$ is an H-matrix with positive diagonals, then for $\|\cdot\|_{p}$ with any $p \geq 1$,

$$
\beta_{p}(M) \leq\left\|\tilde{M}^{-1}\right\|_{p}
$$

where $\tilde{M}$ is the comparison matrix of $M$, that is,

$$
\tilde{M}_{i i}=M_{i i}, \quad \tilde{M}_{i j}=-\left|M_{i j}\right|, \quad \text { for } \quad i \neq j .
$$

- If $M$ is an M-matrix, then for $\|\cdot\|_{p}$ with any $p \geq 1$,

$$
\beta_{p}(M)=\left\|M^{-1}\right\|_{p}
$$

- If $M$ is a symmetric positive definite matrix, then for $\|\cdot\|_{2}$,

$$
\beta_{2}(M)=\left\|M^{-1}\right\|_{2}
$$


Inequalities (1.1) and (1.4) show that the constant $\beta(M)$ derives a new perturbation bound which is sharper than the bound in (i) of Lemma 1.1 in $\|\cdot\|_{\infty}$. Furthermore, Example 2.1 shows that $\beta(M)$ can be much smaller than $c(M)^{-1}$ in some case. Inequality (1.3) indicates that the constant $\beta(M)\|M\|$ is a measure of sensitivity of the solution $x(M, q)$ of the $\operatorname{LCP}(M, q)$. Moreover, from (1.3), (1.6) and (1.7), it is interesting to see that the measure is expressed in the terms of the condition number of $M$, that is,

$$
\kappa_{p}(M):=\left\|M^{-1}\right\|_{p}\|M\|_{p}=\beta_{p}(M)\|M\|_{p}
$$

for $M$ being an M-matrix with $p \geq 1$ and a symmetric positive definite matrix with $p=2$. Hence, it makes connection between perturbation bounds of the LCP and perturbation bounds of the systems of linear equations in the Newton-type methods for solving the LCP. Using the connection, we investigate the efficiency of Newton-type methods for solving the following two systems

$$
r(x):=\min (x, M x+q)=0
$$

and

$$
F(x, y):=\left(\begin{array}{l}
M x+q-y \\
\min (x, y)
\end{array}\right)=0 .
$$

It is known that for the P-matrix LCP, the system of linear equations in Newtontype methods for solving (1.8) or (1.9) is mathematically well-defined, that is, the generalized Jacobian matrices are nonsingular [5]. However, the matrices can be computationally ill-conditioned. A matrix $A$ is said to be an ill-conditioned (wellconditioned) matrix if $\kappa_{p}(A)$ is large (small) [8]. The condition number $\kappa_{p}(A)$ is a measure of sensitivity of the system of linear equations $A x=b$ when $A$ is nonsingular. Hence, a linear system is called ill-conditioned (well-conditioned) if $\kappa_{p}(A)$ is large(small) [4]. From (1.3), (1.6) and (1.7), we find that $\beta_{p}(M)\|M\|_{p}$ is a measure of sensitivety of the $\operatorname{LCP}(M, q)$ when $M$ is a P-matrix, and $\beta_{p}(M)\|M\|_{p}=\kappa_{p}(M)$ when $M$ is an M-matrix or a symmeteric positive definite matrix. Moreover, we show that for the M-matrix LCP, the systems of linear equations in the Newton-type methods for solving (1.8) are well-conditioned if and only if the condition number $\kappa_{p}(M)$ is small. However, the system of linear equations in Newton-type methods for solving (1.9) can be ill-conditioned even when $\kappa_{p}(M)$ is small.

A word about our notation. For a vector $q \in R^{n}, q_{+}=\max (0, q)$. Let $N=$ $\{1,2, \ldots, n\}$. Let $e$ be the vector whose all elements are 1. A matrix $A \in R^{n \times n}$ is called an M-matrix, if $A^{-1} \geq 0$ and $A_{i j} \leq 0(i \neq j)$ for $i, j \in N ; A$ is called an H-matrix, if its comparison matrix is an M-matrix.

In the rest of this paper, we use $\beta(\cdot),\|\cdot\|$ and $\kappa(\cdot)$ to present $\beta_{p}(\cdot),\|\cdot\|_{p}$ and $\kappa_{p}(\cdot)$ with any $p \geq 1$, respectively.

2. A new constant for the P-matrix LCP. In this section we introduce a new Lipschitz constant for the P-matrix LCP based on the observation that for any $x, x^{*}, y, y^{*} \in R^{n}$,

$$
\min \left(x_{i}, y_{i}\right)-\min \left(x_{i}^{*}, y_{i}^{*}\right)=\left(1-d_{i}\right)\left(x_{i}-x_{i}^{*}\right)+d_{i}\left(y_{i}-y_{i}^{*}\right), \quad i \in N
$$

where

$$
d_{i}= \begin{cases}0 & \text { if } y_{i} \geq x_{i}, y_{i}^{*} \geq x_{i}^{*} \\ 1 & \text { if } y_{i} \leq x_{i}, y_{i}^{*} \leq x_{i}^{*} \\ \frac{\min \left(x_{i}, y_{i}\right)-\min \left(x_{i}^{*}, y_{i}^{*}\right)+x_{i}^{*}-x_{i}}{y_{i}-y_{i}^{*}+x_{i}^{*}-x_{i}} & \text { otherwise. }\end{cases}
$$


It is easy to see $d_{i} \in[0,1]$. Set $x=x(A, q), x^{*}=x(B, p), y=A x(A, q)+q, y^{*}=$ $B x(B, p)+p$ in $(2.1)$. We obtain

$$
0=(I-D)(x(A, q)-x(B, p))+D(A x(A, q)+q-B x(B, p)-p)
$$

which implies

$$
(I-D+D A)(x(B, p)-x(A, q))=D(A-B) x(B, p)+D(q-p) .
$$

Here $D$ is a diagonal matrix whose diagonal elements are $d=\left(d_{1}, d_{2}, \ldots, d_{n}\right) \in[0,1]^{n}$.

Lemma 2.1. (Gabriel and Moré[7]) $A$ is a P-matrix if and only if $I-D+D A$ is nonsingular for any diagonal matrix $D=\operatorname{diag}(d)$ with $0 \leq d_{i} \leq 1$.

For $M$ being a P-matrix, we introduce the following constant

$$
\beta(M)=\max _{d \in[0,1]^{n}}\left\|(I-D+D M)^{-1} D\right\| .
$$

From Lemma 2.1 and (2.2), we have

$$
\|x(B, p)-x(A, q)\| \leq \beta(A)\|(A-B) x(B, p)+q-p\|
$$

provided $A$ is a P-matrix. In the following, we compare $\beta(M)$ with $c(M)^{-1}$ in $\|\cdot\|_{\infty}$ and give a simple version of $\beta(M)$ for $M$ being an M-matrix, a symmetric positive definite matrix, and positive definite matrix.

Theorem 2.2. Let $M$ be a P-matrix. Then

$$
\beta_{\infty}(M):=\max _{d \in[0,1]^{n}}\left\|(I-D+D M)^{-1} D\right\|_{\infty} \leq \frac{1}{c(M)} .
$$

Proof. We first prove that for any nonsingular diagonal matrix $D=\operatorname{diag}(d)$ with $d \in(0,1]^{n}$,

$$
\left\|(I-D+D M)^{-1} D\right\|_{\infty} \leq \frac{1}{c(M)} .
$$

Let $x \in R^{n}$ with $\|x\|_{\infty}=1$ such that $\left\|(I-D+D M)^{-1} D\right\|_{\infty}=\left\|(I-D+D M)^{-1} D x\right\|_{\infty}$ and define $y=(I-D+D M)^{-1} D x$. Then $D x=(I-D+D M) y, M y=x+y-D^{-1} y$. By the definition of $c(M)$, we have

$$
0<c(M)\|y\|_{\infty}^{2} \leq \max _{i} y_{i}(M y)_{i}=\max _{i} y_{i}\left(x_{i}+y_{i}-\frac{y_{i}}{d_{i}}\right) .
$$

Note that $f(t)=a\left(b+a-\frac{a}{t}\right)$ is monotonically increasing for $t>0$, where $a, b$ are constants. Therefore, we deduce

$$
y_{i}\left(x_{i}+y_{i}-\frac{y_{i}}{d_{i}}\right) \leq y_{i} x_{i} \leq\|y\|_{\infty}\|x\|_{\infty}=\|y\|_{\infty},
$$

which implies

$$
0<c(M)\|y\|_{\infty}^{2} \leq\|y\|_{\infty} \quad \text { and } \quad\left\|(I-D+D M)^{-1} D\right\|_{\infty}=\|y\|_{\infty} \leq \frac{1}{c(M)} .
$$


Now we consider $d \in[0,1]^{n}$. Let $d_{\epsilon}=\min (d+\epsilon e, e)$, where $\epsilon \in(0,1]$. Then, we have

$$
\left\|(I-D+D M)^{-1} D\right\|_{\infty}=\lim _{\epsilon \downarrow 0}\left\|\left(I-D_{\epsilon}+D_{\epsilon} M\right)^{-1} D_{\epsilon}\right\|_{\infty} \leq \frac{1}{c(M)} .
$$

口

It is known that an $\mathrm{H}$-matrix with positive diagonals is a $\mathrm{P}$-matrix, and a positive definite matrix is a P-matrix [3]. Now, we consider the two subclasses of P-matrix.

Lemma 2.3. ([3]) If $M$ is an $M$-matrix, then $I-D+D M$ is an $M$-matrix for $d \in[0,1]^{n}$.

Lemma 2.4. Let $A$ be an H-matrix with positive diagonals, and let $\tilde{A}$ be the comparison matrix of $A$. Then the following statements hold:

(i) $\left|A^{-1}\right| \leq \tilde{A}^{-1}$.

(ii) For $B \in R^{n \times n}$ with $\|B\|_{\infty}\left\|\tilde{A}^{-1}\right\|_{\infty}<1, A+B$ is an H-matrix with positive diagonals.

Proof. (i) See problem 31 in [10, page 131]

(ii) Let $x=\tilde{A}^{-1} e$. Since $\tilde{A}^{-1} \geq 0, x>0$ and $\|x\|_{\infty}=\left\|\tilde{A}^{-1}\right\|_{\infty}$. Moreover, from $\tilde{A} x=e$, we have

$$
a_{i i} x_{i}=1+\sum_{j \neq i}\left|a_{i j}\right| x_{j}, \quad \text { for } i \in N .
$$

By $\|x\|_{\infty}\|B\|_{\infty}<1$ and $\|B\|_{\infty}=\||B| e\|_{\infty}$, we get $\|x\|_{\infty}|B| e<e$. Hence for all $i \in N$,

$$
a_{i i} x_{i}>\sum_{j=1}^{n}\left|b_{i j}\right|\left|x \|_{\infty}+\sum_{j \neq i}\right| a_{i j}\left|x_{j} \geq \sum_{j \neq i}\left(\left|a_{i j}\right|+\left|b_{i j}\right|\right) x_{j}+\right| b_{i i} \mid x_{i},
$$

and

$$
\left(a_{i i}+b_{i i}\right) x_{i} \geq\left(a_{i i}-\left|b_{i i}\right|\right) x_{i}>\sum_{j \neq i}\left(\left|a_{i j}\right|+\left|b_{i j}\right|\right) x_{j} .
$$

By $\mathrm{I}_{27}$ of Theorem 2.3, Chap. 6 in [1], this implies that the comparison matrix of $A+B$ is an M-matrix. Hence $A+B$ is an H-matrix with positive diagonals. $\square$

Theorem 2.5. Let $M$ be an H-matrix with positive diagonals. Then

$$
\beta(M) \leq\left\|\tilde{M}^{-1}\right\|
$$

where $\tilde{M}$ is the comparison matrix of $M$. In particular, if $M$ is an $M$-matrix, then the equality holds with $M=\tilde{M}$.

Proof. First we will show that if $M$ is an M-matrix, then

$$
\beta(M)=\left\|M^{-1}\right\|
$$

Since for any $d \in(0,1]^{n}$, by Lemma 2.3 ,

$$
(D M)^{-1}-(I-D+D M)^{-1}=(D M)^{-1}(I-D)(I-D+D M)^{-1} \geq 0,
$$

we have

$$
(D M)^{-1} D-(I-D+D M)^{-1} D=M^{-1}-(I-D+D M)^{-1} D \geq 0 .
$$


Note that for any matrices $A$ and $B,|A| \leq B$ implies $\|A\| \leq\|B\|$. Hence the following inequalities hold

$$
M^{-1} \geq(I-D+D M)^{-1} D \geq 0, \quad\left\|M^{-1}\right\| \geq\left\|(I-D+D M)^{-1} D\right\| .
$$

Let $d_{\epsilon}=\min (d+\epsilon e, e)$, where $\epsilon \in(0,1]$. Then, we have

$$
\beta(M)=\max _{d \in[0,1]^{n}} \lim _{\epsilon \downarrow 0}\left\|\left(I-D_{\epsilon}+D_{\epsilon} M\right)^{-1} D_{\epsilon}\right\| \leq\left\|M^{-1}\right\| .
$$

It is obvious that $\beta(M) \geq\left\|M^{-1}\right\|$ as $e \in[0,1]^{n}$. Therefore, we obtain $\beta(M)=\left\|M^{-1}\right\|$.

For $M$ being an H-matrix, $\tilde{M}$ is an M-matrix. From (i) of Lemma 2.4, we have

$$
\left|(I-D+D M)^{-1}\right| \leq(I-D+D \tilde{M})^{-1} .
$$

Hence, we obtain

$$
\beta(M)=\max _{d \in[0,1]^{n}}\left\|(I-D+D M)^{-1} D\right\| \leq \max _{d \in[0,1]^{n}}\left\|(I-D+D \tilde{M})^{-1} D\right\| \leq\left\|\tilde{M}^{-1}\right\| .
$$

口

Lemma 2.6. [9] Let $A$ and $B$ be symmetric positive definite matrices.

(i) $B-A$ is positive semidefinite if and only if $A^{-1}-B^{-1}$ is positive semidefinite.

(ii) If $B-A$ is positive semidefinite, then $\lambda_{i}(B) \geq \lambda_{i}(A)$, where $\lambda_{1}(A) \geq \lambda_{2}(A) \geq$ $\cdots \geq \lambda_{n}(A)$ and $\lambda_{1}(B) \geq \lambda_{2}(B) \geq \cdots \geq \lambda_{n}(B)$ are eigenvalues of $A$ and $B$, respectively.

THEOREM 2.7. Let $M$ be a symmetric positive definite matrix. Then

$$
\beta_{2}(M):=\max _{d \in[0,1]^{n}}\left\|(I-D+D M)^{-1} D\right\|_{2}=\left\|M^{-1}\right\|_{2} .
$$

Proof. It is obvious that $\beta_{2}(M) \geq\left\|M^{-1}\right\|_{2}$. Now we show $\beta_{2}(M) \leq\left\|M^{-1}\right\|_{2}$. For any nonsingular diagonal matrix $D=\operatorname{diag}(d)$ with $d \in(0,1]^{n}, M+D^{-1}(I-D)$ is positive definite. By (i) of Lemma 2.6, $M^{-1}-\left(M+D^{-1}(I-D)\right)^{-1}$ is positive semidefinite. By (ii) of Lemma 2.6, we have

$$
\left\|\left(M+D^{-1}(I-D)\right)^{-1}\right\|_{2}=\left\|(I-D+D M)^{-1} D\right\|_{2} \leq\left\|M^{-1}\right\|_{2} .
$$

Since the largest eigenvalue is a continuous function of elements of the matrix, we have

$$
\beta_{2}(M)=\max _{d \in[0,1]^{n}} \lim _{\epsilon \downarrow 0}\left\|\left(I-D_{\epsilon}+D_{\epsilon} M\right)^{-1} D_{\epsilon}\right\|_{2} \leq\left\|M^{-1}\right\|_{2},
$$

where $D_{\epsilon}=\operatorname{diag}(\min (d+\epsilon e, e))$.

In comparison to Lemma 1.1, the following theorem gives sharp perturbation error estimates for the P-matrix LCP

Theorem 2.8. Let $M \in R^{n \times n}$ be a P-matrix. Then the following statements hold:

(i) For any two vectors $q$ and $p$ in $R^{n}$,

$$
\|x(M, q)-x(M, p)\| \leq \beta(M)\|q-p\| .
$$


(ii) Every matrix $A \in \mathcal{M}:=\{A \mid \beta(M)\|M-A\| \leq \eta<1\}$ is a P-matrix. Let

$$
\alpha(M)=\frac{1}{1-\eta} \beta(M) .
$$

Then for any $A, B \in \mathcal{M}$ and $q, p \in R^{n}$

$$
\|x(A, q)-x(B, p)\| \leq \alpha(M)^{2}\left\|(-p)_{+}\right\|\|A-B\|+\alpha(M)\|q-p\| .
$$

Proof. (i) It follows directly from (2.3) by setting $M=A=B$.

(ii) For every $A \in \mathcal{M}$, since $\left\|(I-D+D M)^{-1} D(A-M)\right\| \leq \beta(M)\|M-A\| \leq \eta<1$,

$$
(I-D+D A)=(I-D+D M)\left(I+(I-D+D M)^{-1} D(A-M)\right)
$$

is nonsingular for any diagonal matrix $D=\operatorname{diag}(d)$ with $0 \leq d_{i} \leq 1$. By Lemma 2.1, $A$ is a $\mathrm{P}$-matrix. Moreover, from

$$
(I-D+D A)^{-1} D=\left(I+(I-D+D M)^{-1} D(A-M)\right)^{-1}(I-D+D M)^{-1} D,
$$

and

$$
\left\|\left(I+(I-D+D M)^{-1} D(A-M)\right)^{-1}\right\| \leq \frac{1}{1-\beta(M)\|A-M\|} \leq \frac{1}{1-\eta},
$$

we find $\beta(A) \leq \alpha(M)$.

Since matrices $A, B \in \mathcal{M}$ are P-matrices, using (2.3) yields,

$$
\|x(A, q)-x(B, p)\| \leq \beta(A)(\|A-B\|\|x(B, p)\|+\|q-p\|) .
$$

Notice that 0 is the solution of $\operatorname{LCP}\left(B, p_{+}\right)$. Using (2.3) again, we get

$$
\|x(B, p)\| \leq \beta(B)\left\|(-p)_{+}\right\| .
$$

Applying $\beta(A) \leq \alpha(M)$ and $\beta(B) \leq \alpha(M)$ to (2.4) and (2.5), respectively, we obtain the desired bounds in (ii). $\square$

From Theorem 2.5 and Theorem 2.7, the Lipschitz constants $\beta(M)$ and $\alpha(M)$ can be estimated by matrix norms, if $M$ is an H-matrix with positive diagonals or a symmetric positive definite matrix. In particular, from Lemma 2.4, Theorem 2.5 and Theorem 2.7, we have the following two corollaries.

Corollary 2.9. Let $M \in R^{n \times n}$ be an H-matrix with positive diagonals. Then the following statements hold:

(i) For any two vectors $q$ and $p$ in $R^{n}$,

$$
\|x(M, q)-x(M, p)\|_{\infty} \leq\left\|\tilde{M}^{-1}\right\|_{\infty}\|q-p\|_{\infty}
$$

(ii) Every matrix $A \in \mathcal{M}_{\infty}:=\left\{A \mid\left\|\tilde{M}^{-1}\right\|_{\infty}\|M-A\|_{\infty} \leq \eta<1\right\}$ is an H-matrix with positive diagonals. Let

$$
\alpha_{\infty}(M)=\frac{1}{1-\eta}\left\|\tilde{M}^{-1}\right\|_{\infty}
$$

Then for any $A, B \in \mathcal{M}_{\infty}$ and $q, p \in R^{n}$

$$
\|x(A, q)-x(B, p)\|_{\infty} \leq \alpha_{\infty}(M)^{2}\left\|(-p)_{+}\right\|_{\infty}\|A-B\|_{\infty}+\alpha_{\infty}(M)\|q-p\|_{\infty} .
$$


COROllary 2.10. Let $M \in R^{n \times n}$ be a symmetric positive definite matrix. Then the following statements hold:

(i) For any two vectors $q$ and $p$ in $R^{n}$,

$$
\|x(M, q)-x(M, p)\|_{2} \leq\left\|M^{-1}\right\|_{2}\|q-p\|_{2}
$$

(ii) Every matrix $A \in \mathcal{M}_{2}:=\left\{A \mid\left\|M^{-1}\right\|_{2}\|M-A\|_{2} \leq \eta<1\right\}$ is a P-matrix. Let

$$
\alpha_{2}(M)=\frac{1}{1-\eta}\left\|M^{-1}\right\|_{2}
$$

Then for any $A, B \in \mathcal{M}_{2}$ and $q, p \in R^{n}$

$$
\|x(A, q)-x(B, p)\|_{2} \leq \alpha_{2}(M)^{2}\left\|(-p)_{+}\right\|_{2}\|A-B\|_{2}+\alpha_{2}(M)\|q-p\|_{2} .
$$

A matrix $A$ is called positive definite if

$$
x^{T} A x>0, \quad 0 \neq x \in R^{n} .
$$

Since $x^{T} A x=x^{T} \frac{A+A^{T}}{2} x, A$ is positive definite if and only if $\frac{A+A^{T}}{2}$ is symmetric positive definite. Note that a positive definite matrix is not necessarily symmetric. Such asymmetric matrices frequently appear in the context of the LCP.

Combining the ideas of Mathias and Pang [12] and Corollary 2.10, we present perturbation bounds for the positive definite matrix LCP.

TheOREM 2.11. Let $M \in R^{n \times n}$ be a positive definite matrix. Then the following statements hold:

(i) For any two vectors $q$ and $p$ in $R^{n}$,

$$
\|x(M, q)-x(M, p)\|_{2} \leq\left\|\left(\frac{M+M^{T}}{2}\right)^{-1}\right\|_{2}\|q-p\|_{2}
$$

(ii) Every matrix $A \in \mathcal{M}_{2}:=\left\{A \mid\left\|\left(\frac{M+M^{T}}{2}\right)^{-1}\right\|_{2}\|M-A\|_{2} \leq \eta<1\right\}$ is positive definite. Let

$$
\alpha_{2}(M)=\frac{1}{1-\eta}\left\|\left(\frac{M+M^{T}}{2}\right)^{-1}\right\|_{2}
$$

Then for any $A, B \in \mathcal{M}_{2}$ and $q, p \in R^{n}$

$$
\|x(A, q)-x(B, p)\|_{2} \leq \alpha_{2}(M)^{2}\left\|(-p)_{+}\right\|_{2}\|A-B\|_{2}+\alpha_{2}(M)\|q-p\|_{2} .
$$

Proof. We first show that the following inequality holds

$$
\|x(A, q)-x(B, p)\|_{2} \leq\left\|\left(\frac{A+A^{T}}{2}\right)^{-1}\right\|_{2}\left(\|A-B\|_{2}\|x(B, p)\|_{2}+\|p-q\|_{2}\right),
$$

if $A$ is a positive definite matrix and the $\operatorname{LCP}(B, p)$ has a solution $x(B, p)$.

Since $x(A, q)$ and $x(B, p)$ are solutions of the $\operatorname{LCP}(A, q)$ and $\operatorname{LCP}(B, p)$, respectively, we have

$$
\begin{aligned}
0 & \geq(x(A, q)-x(B, p))^{T}(A x(A, q)+q-B x(B, p)-p) \\
& =(x(A, q)-x(B, p))^{T}(A(x(A, q)-x(B, p))+(A-B) x(B, p)+q-p),
\end{aligned}
$$


which implies

$$
\begin{aligned}
& (x(A, q)-x(B, p))^{T}((B-A) x(B, p)+p-q) \\
\geq & (x(A, q)-x(B, p))^{T} A(x(A, q)-x(B, p)) \\
= & (x(A, q)-x(B, p))^{T} \frac{A+A^{T}}{2}(x(A, q)-x(B, p)) \\
\geq & \frac{1}{\left\|\left(\frac{A+A^{T}}{2}\right)^{-1}\right\|_{2}}\|x(A, q)-x(B, p)\|_{2}^{2} .
\end{aligned}
$$

Using the Cauchy-Schwartz inequality, we get (2.6).

(i) Set $A=B=M$ in (2.6), we get the desired bound.

(ii) Note that for any matrix $C,\|C\|_{2}=\left\|C^{T}\right\|_{2}$. For any $x \in R^{n}$ with $x \neq 0$, we have

$$
\begin{aligned}
x^{T} A x & =x^{T} \frac{M+M^{T}}{2} x+x^{T}\left(\frac{A+A^{T}}{2}-\frac{M+M^{T}}{2}\right) x \\
& \geq x^{T} \frac{M+M^{T}}{2} x-\left(\left\|\frac{A-M}{2}\right\|_{2}+\left\|\frac{A^{T}-M^{T}}{2}\right\|_{2}\right)\|x\|_{2}^{2} \\
& \geq\left(\left\|\left(\frac{M+M^{T}}{2}\right)^{-1}\right\|_{2}\right)^{-1}\|x\|_{2}^{2}-\|A-M\|_{2}\|x\|_{2}^{2} \\
& \geq\left(\left\|\left(\frac{M+M^{T}}{2}\right)^{-1}\right\|_{2}\right)^{-1}\left(1-\left\|\left(\frac{M+M^{T}}{2}\right)^{-1}\right\|_{2}\|M-A\|_{2}\right)\|x\|_{2}^{2} .
\end{aligned}
$$

Hence for any $A \in \mathcal{M}_{2}, x^{T} A x>0$, and thus $A$ is positive definite. Moreover, from

$$
\left(\frac{A+A^{T}}{2}\right)^{-1}=\left(I+\left(\frac{M+M^{T}}{2}\right)^{-1}\left(\frac{A+A^{T}}{2}-\frac{M+M^{T}}{2}\right)\right)^{-1}\left(\frac{M+M^{T}}{2}\right)^{-1}
$$

and

$$
\left\|\frac{A+A^{T}}{2}-\frac{M+M^{T}}{2}\right\|_{2} \leq \frac{1}{2}\left(\|A-M\|_{2}+\left\|A^{T}-M^{T}\right\|_{2}\right)=\|M-A\|_{2}
$$

we have

$\left\|\left(\frac{A+A^{T}}{2}\right)^{-1}\right\|_{2} \leq \frac{1}{1-\left\|\left(\frac{M+M^{T}}{2}\right)^{-1}\right\|_{2}\left\|\frac{A+A^{T}}{2}-\frac{M+M^{T}}{2}\right\|_{2}}\left\|\left(\frac{M+M^{T}}{2}\right)^{-1}\right\|_{2} \leq \alpha_{2}(M)$.

Similarly, for $B \in \mathcal{M}_{2},\left\|\left(\frac{B+B^{T}}{2}\right)^{-1}\right\|_{2} \leq \alpha_{2}(M)$. Notice that 0 is the solution of $\operatorname{LCP}\left(B, p_{+}\right)$. Setting $A=B$ and $q=p_{+}$in (2.6), we get

$$
\|x(B, p)\|_{2} \leq\left\|\left(\frac{B+B^{T}}{2}\right)^{-1}\right\|_{2}\left\|(-p)_{+}\right\|_{2} .
$$

Using these inequalities with (2.6), we obtain the perturbation bound in (ii). $\square$

Example 2.1 Theorem 2.2 shows that for every P-matrix, $\beta_{\infty}(M) \leq c(M)^{-1}$. Now we show that $\beta_{\infty}(M)$ can be much smaller than $c(M)^{-1}$ in some case. Consider

$$
M=\left(\begin{array}{rr}
1 & -t \\
0 & t
\end{array}\right) \text {. }
$$

For $t \geq 1, M$ is an M-matrix. By Theorem 2.5, $\beta_{\infty}(M)=\left\|M^{-1}\right\|_{\infty}=2$. For $\bar{x}=\left(1, t^{-1}\right)$, we have

$$
c(M) \leq \max _{i \in N} \bar{x}_{i}(M \bar{x})_{i}=\frac{1}{t} .
$$

Hence, $c(M)^{-1} \geq t \rightarrow \infty$, as $t \rightarrow \infty$. 
3. Relative perturbation bounds for the LCP. Using the results in the last section, we derive relative perturbation bounds expressed in the term of $\beta(M)\|M\|$.

THEOREM 3.1. Suppose

$$
\begin{aligned}
& \min (x, M x+q)=0 \quad M \in R^{n \times n}, \quad 0 \neq(-q)_{+} \in R^{n} \\
& \min (y,(M+\triangle M) y+q+\triangle q) \quad=0 \quad \triangle M \in R^{n \times n}, \quad \triangle q \in R^{n}
\end{aligned}
$$

with

$$
\|\triangle M\| \leq \epsilon\|M\|, \quad\|\triangle q\| \leq \epsilon\left\|(-q)_{+}\right\| .
$$

If $M$ is a P-matrix and $\epsilon \beta(M)\|M\|=\eta<1$, then $M+\triangle M$ is a P-matrix and

$$
\frac{\|y-x\|}{\|x\|} \leq \frac{2 \epsilon}{1-\eta} \beta(M)\|M\| \text {. }
$$

Proof. First we observe that $x$ is a solution of $\operatorname{LCP}(M, q)$ and $y$ is a solution of $\mathrm{LCP}(M+\triangle M, q+\triangle q)$. Then following the proof of (ii) of Theorem 2.8, we obtain that $M+\triangle M$ is a $\mathrm{P}$-matrix and

$$
\beta(M+\triangle M) \leq \frac{1}{1-\eta} \beta(M),
$$

which, together with (2.3), give

$$
\|x-y\| \leq \frac{1}{1-\eta} \beta(M)(\|\triangle M\|\|x\|+\|\triangle q\|) .
$$

From $M x+q \geq 0$, we deduce $(-q)_{+} \leq(M x)_{+} \leq|M x|$. This implies $\left\|(-q)_{+}\right\| \leq$ $\|M x\| \leq\|M\|\|x\|$. Hence, we have

$$
\|x\| \geq \frac{1}{\|M\|}\left\|(-q)_{+}\right\|>0
$$

Combining (3.1) and (3.2), we obtain the desired bounds

$$
\frac{\|y-x\|}{\|x\|} \leq \frac{1}{1-\eta} \beta(M)\left(\|\triangle M\|+\frac{\|\triangle q\|}{\|x\|}\right) \leq \frac{2 \epsilon}{1-\eta} \beta(M)\|M\| .
$$

Theorem 3.1 indicates that $\beta(M)\|M\|$ is a measure of sensitivity of the solution of the $\operatorname{LCP}(M, q)$ for $M$ being a P-matrix. Moreover, Theorem 3.1 with Corollary 2.9, Corollary 2.10 and Theorem 2.11 gives $\beta(M)\|M\|$ in the term of condition number for the H-matrix LCP, symmetric positive definite LCP and positive definite LCP.

Corollary 3.2. Suppose

$$
\begin{aligned}
& \min (x, M x+q)=0 \quad M \in R^{n \times n}, \quad 0 \neq(-q)_{+} \in R^{n} \\
& \min (y,(M+\triangle M) y+q+\triangle q) \quad=0 \quad \triangle M \in R^{n \times n}, \quad \triangle q \in R^{n} \text {. }
\end{aligned}
$$

(i) If $M$ is an H-matrix with positive diagonals, $\epsilon \kappa_{\infty}(\tilde{M})=\eta<1$, and

$$
\|\triangle M\|_{\infty} \leq \epsilon\|\tilde{M}\|_{\infty}, \quad\|\triangle q\|_{\infty} \leq \epsilon\left\|(-q)_{+}\right\|_{\infty}
$$

then $M+\triangle M$ is an $H$-matrix with positive diagonals and

$$
\frac{\|y-x\|_{\infty}}{\|x\|_{\infty}} \leq \frac{2 \epsilon}{1-\eta} \kappa_{\infty}(\tilde{M})
$$


(ii) If $M$ is a symmetric positive definite matrix, $\epsilon \kappa_{2}(M)=\eta<1$, and

$$
\|\triangle M\|_{2} \leq \epsilon\|M\|_{2}, \quad\|\triangle q\|_{2} \leq \epsilon\left\|(-q)_{+}\right\|_{2},
$$

then $M+\triangle M$ is a P-matrix and

$$
\frac{\|y-x\|_{2}}{\|x\|_{2}} \leq \frac{2 \epsilon}{1-\eta} \kappa_{2}(M) .
$$

(iii) If $M$ is a positive definite matrix, $\epsilon \kappa_{2}\left(\frac{M+M^{T}}{2}\right)=\eta<1$, and

$$
\|\triangle M\|_{2} \leq \epsilon\left\|\frac{M+M^{T}}{2}\right\|_{2}, \quad\|\triangle q\|_{2} \leq \epsilon\left\|(-q)_{+}\right\|_{2} \frac{\left\|M+M^{T}\right\|_{2}}{2\|M\|_{2}},
$$

then $M+\triangle M$ is a positive definite matrix, and

$$
\frac{\|x-y\|_{2}}{\|x\|_{2}} \leq \frac{2 \epsilon}{1-\eta} \kappa_{2}\left(\frac{M+M^{T}}{2}\right) .
$$

Remark Note that $\left\|(-q)_{+}\right\| \leq\|q\|$. If $M x+q=0$, then (i) of Corollary 3.2 for $M$ being an M-matrix and (ii) of Corollary 3.2 reduce to the perturbation bounds for the system of linear equations [8].

For the H-matrix LCP, componentwise perturbation bounds based on the Skeel condition number $\left\|\left|\tilde{M}^{-1}\|\tilde{M} \mid\|_{\infty}\right.\right.$ can be represented as follows.

Theorem 3.3. Suppose

$$
\begin{aligned}
& \min (x, M x+q)=0 \quad M \in R^{n \times n}, \quad 0 \neq(-q)_{+} \in R^{n} \\
& \min (y,(M+\triangle M) y+q+\triangle q)=0 \quad \triangle M \in R^{n \times n}, \quad \triangle q \in R^{n}
\end{aligned}
$$

with

$$
|\triangle M| \leq \epsilon|M|, \quad|\triangle q| \leq \epsilon(-q)_{+}
$$

If $M$ is an H-matrix with positive diagonals and $\epsilon \kappa_{\infty}(\tilde{M})=\eta<1$, then $M+\triangle M$ is an H-matrix with positive diagonals and

$$
\frac{\|y-x\|_{\infty}}{\|x\|_{\infty}} \leq \frac{2 \epsilon}{1-\eta}\left\|\tilde{M}^{-1}|\tilde{M}|\right\|_{\infty}
$$

Proof. From (3.3), we have

$$
\|\triangle M\|_{\infty} \leq \epsilon\|\tilde{M}\|_{\infty}, \quad \text { and } \quad\|\triangle q\|_{\infty} \leq \epsilon\left\|(-q)_{+}\right\|_{\infty}, \leq \epsilon\|M\|_{\infty}\|x\|_{\infty},
$$

where the last inequality uses $(-q)_{+} \leq(M x)_{+} \leq|M| x$.

According to Corollary $3.2, M+\triangle M$ is an H-matrix with positive diagonals. Moreover, the equality (2.2) gives

$$
(I-D+D M)(y-x)=D \triangle M y+D \triangle q,
$$

for some diagonal matrix $D=\operatorname{diag}(d)$ with $d \in[0,1]^{n}$.

Following the proof of Theorem 2.5, by Lemma 2.4, we get

$$
\begin{aligned}
|y-x| & \leq\left|(I-D+D M)^{-1} D\right|(|\triangle M| y+|\triangle q|) \\
& \leq\left|(I-D+D \tilde{M})^{-1} D\right|(|\triangle M| y+|\triangle q|) \\
& \leq \tilde{M}^{-1}(|\triangle M| y+|\triangle q|) \\
& \leq \epsilon \tilde{M}^{-1}(|M| y+|M| x) .
\end{aligned}
$$


Therefore, we find

$$
\|y-x\|_{\infty} \leq \epsilon\left\|\tilde{M}^{-1}|M|\right\|_{\infty}\left(\|y\|_{\infty}+\|x\|_{\infty}\right) .
$$

Furthermore, from (3.5), we obtain

$$
y-\left((I-D+D M)^{-1} D \triangle M\right) y=x+(I-D+D M)^{-1} D \triangle q .
$$

Hence, it holds

$$
\begin{aligned}
\left(1-\epsilon\left\|\tilde{M}^{-1}\right\|_{\infty}\|M\|_{\infty}\right)\|y\|_{\infty} & \leq\left(1-\left\|(I-D+D M)^{-1} D\right\|_{\infty}\|\triangle M\|_{\infty}\right)\|y\|_{\infty} \\
& \leq\left\|y-(I-D+D M)^{-1} D \triangle M y\right\|_{\infty} \\
& \left.\leq\|x\|_{\infty}+\| I-D+D M\right)^{-1} D\left\|_{\infty}\right\| \triangle q \|_{\infty} \\
& \leq\left(1+\epsilon\left\|\tilde{M}^{-1}\right\|_{\infty}\|M\|_{\infty}\right)\|x\|_{\infty} .
\end{aligned}
$$

This implies

$$
\|y\|_{\infty} \leq \frac{1+\eta}{1-\eta}\|x\|_{\infty}
$$

Combining (3.6) and (3.7), we obtain the desired bounds (3.4).

4. Newton-type methods. In the last two sections, we have given perturbation bounds for the LCP in the term of $\beta(M)$. In this section, we use the perturbation bounds to analyze efficiency of Newton-type methods for solving the LCP based on the systems (1.8) and (1.9).

Many semismooth Newton methods, smoothing Newton methods and path-following interior point methods [5] solve a system of linear equations in their each iteration,

$$
\left(I-D_{k}+D_{k} M\right)\left(x-x^{k}\right)=-r\left(x^{k}\right),
$$

or

$$
\left(\begin{array}{cc}
M & -I \\
I-D_{k} & D_{k}
\end{array}\right)\left(\begin{array}{c}
x-x^{k} \\
y-y^{k}
\end{array}\right)=-F\left(x^{k}, y^{k}\right)
$$

where $D_{k}$ is a diagonal matrix whose diagonal elements are in $[0,1]$.

Sensitivity of (4.1) and (4.2) will effect implementation of the methods and reliability of the computed solution. From the analysis of Dennis and Schnabel[4], if the condition number of the coefficient matrix of the linear equations is larger than (macheps $)^{-1 / 2}$, the numerical solution may not be trustworthy. Here macheps is computer precision. The linear systems (4.1) and (4.2) have the following relation regarding to the condition numbers.

Proposition 4.1. For any diagonal matrix $D=\operatorname{diag}(d)$ with $0 \leq d_{i} \leq 1, i=$ $1,2, \ldots, n$, the following inequalities hold

$$
\kappa_{\infty}\left(\begin{array}{cc}
M & -I \\
I-D & D
\end{array}\right) \geq \kappa_{\infty}(I-D+D M)
$$

and

$$
\kappa\left(\begin{array}{cc}
M & -I \\
I-D & D
\end{array}\right) \geq \frac{1}{2} \kappa(I-D+D M) .
$$


Proof. First we observe

$$
\left\|\left(\begin{array}{cc}
M & -I \\
I-D & D
\end{array}\right)\right\|_{\infty} \geq 1+\|M\|_{\infty} \geq \max \left(1,\|M\|_{\infty}\right) \geq\|I-D+D M\|_{\infty}
$$

and

$\left\|\left(\begin{array}{cc}M & -I \\ I-D & D\end{array}\right)\right\| \geq \max (1,\|M\|) \geq \frac{\max (1,\|M\|)}{1+\|M\|}\|I-D+D M\| \geq \frac{1}{2}\|I-D+D M\|$.

Next, we consider the inverses. From

$$
\left(\begin{array}{cc}
I & 0 \\
D & I
\end{array}\right)\left(\begin{array}{cc}
M & -I \\
I-D & D
\end{array}\right)=\left(\begin{array}{cc}
M & -I \\
I-D+D M & 0
\end{array}\right)
$$

and

$$
\left(\begin{array}{cc}
M & -I \\
I-D+D M & 0
\end{array}\right)^{-1}=\left(\begin{array}{cc}
0 & (I-D+D M)^{-1} \\
-I & M(I-D+D M)^{-1}
\end{array}\right)
$$

we find the inverse

$$
\begin{aligned}
\left(\begin{array}{cc}
M & -I \\
I-D & D
\end{array}\right)^{-1} & =\left(\begin{array}{cc}
0 & (I-D+D M)^{-1} \\
-I & M(I-D+D M)^{-1}
\end{array}\right)\left(\begin{array}{cc}
I & 0 \\
D & I
\end{array}\right) \\
& =\left(\begin{array}{cc}
(I-D+D M)^{-1} D & (I-D+D M)^{-1} \\
M(I-D+D M)^{-1} D-I & M(I-D+D M)^{-1}
\end{array}\right)
\end{aligned}
$$

Therefore, we have

$$
\left\|\left(\begin{array}{cc}
M & -I \\
I-D & D
\end{array}\right)^{-1}\right\| \geq\left\|(I-D+D M)^{-1}\right\| .
$$

By the definition of the condition number, (4.3) and (4.4) hold.

Since $D_{k}$ in the coefficient matrices of (4.1) and (4.2) changes at each step, we consider the worst case

$$
K(M):=\max _{d \in[0,1]^{n}}\left\|(I-D+D M)^{-1}\right\|\|I-D+D M\|
$$

and

$$
\hat{K}(M):=\max _{d \in[0,1]^{n}}\left\|\left(\begin{array}{cc}
M & -I \\
D & I-D
\end{array}\right)^{-1}\right\|\left\|\left(\begin{array}{cc}
M & -I \\
D & I-D
\end{array}\right)\right\| .
$$

From Proposition 4.1, we have

$$
\hat{K}_{\infty}(M) \geq K_{\infty}(M)
$$

which implies that if (4.2) is well-conditioned, then (4.1) is well-conditioned, and if (4.1) is ill-conditioned, then (4.2) is ill-conditioned. The following example shows that $\hat{K}_{\infty}(M)$ can be much larger than $K_{\infty}(M)$.

Example 4.1 Let $M=a I(a \geq 1)$. Straightforward calculation gives

$$
\hat{K}_{\infty}(M) \geq\left\|\left(\begin{array}{cc}
a I & -I \\
I & 0
\end{array}\right)\right\|_{\infty}\left\|\left(\begin{array}{cc}
a I & -I \\
I & 0
\end{array}\right)^{-1}\right\|_{\infty}=(1+a)\left\|\left(\begin{array}{cc}
0 & I \\
-I & a I
\end{array}\right)\right\|_{\infty}=(1+a)^{2}
$$


and

$K_{\infty}(M)=\max _{d \in[0,1]^{n}}\left\|(I-D+a D)^{-1}\right\|_{\infty}\|I-D+a D\|_{\infty} \leq \frac{\max _{0 \leq \xi \leq 1}|(1+a \xi-\xi)|}{\min _{0 \leq \xi \leq 1}|(1+a \xi-\xi)|}=a$.

For large $a, \hat{K}_{\infty}(M)-K_{\infty}(M)\left(\geq a^{2}+a+1\right)$ is very large.

From Proposition 4.1 and Example 4.1, we may suggest Newton-type methods for solving the nonlinear equations (1.8) have less perturbation error than the Newtontype methods for (1.9). Now, we focus on Newton-type methods for (1.8). Obviously, it holds

$$
K(M) \geq \kappa(M),
$$

as $e \in[0,1]^{n}$. For $M$ being an H-matrix with positive diagonals, by Theorem 2.1 and Theorem 2.3 in [2], we have

$$
K_{\infty}(M) \leq \max \left(1,\|M\|_{\infty}\right)\left\|\tilde{M}^{-1} \max (\Lambda, I)\right\|_{\infty},
$$

where $\Lambda$ is the diagonal parts of $M$.

For $M$ being an M-matrix with $\|M\|_{\infty} \geq 1$, we have

$$
\kappa_{\infty}(M) \leq K_{\infty}(M) \leq \kappa_{\infty}(M)\|\max (\Lambda, I)\|_{\infty} .
$$

Hence, the condition number $\kappa_{\infty}(M)$ is a measure of sensitivity of the solution of the system of linear equations for the worst case. Note that we have shown that $\kappa_{\infty}(M)$ is a measure of sensitivity of the solution of LCP. Hence we may suggest that if $\Lambda$ is not large, then the LCP is well-conditioned if and only if the system of linear equations (4.1) at each step of the Newton method is well-conditioned. Furthermore, for an $M$ matrix, its diagonal elements are positive, and the $\operatorname{LCP}\left(\Lambda^{-1} M, \Lambda^{-1} q\right)$ and $\operatorname{LCP}(M, q)$ are equivalent. The inequalities in (4.6) yield $K_{\infty}\left(\Lambda^{-1} M\right)=\kappa_{\infty}\left(\Lambda^{-1} M\right)$.

5. Final remark. In [2], we provided the following error bound for the P-matrix LCP

$$
\|x-x(M, q)\| \leq \max _{d \in[0,1]^{n}}\left\|(I-D+D M)^{-1}\right\|\|r(x)\|, \quad \text { for any } x \in R^{n}
$$

and proved that (5.1) is sharper than the Mathias-Pang error bound [12]

$$
\|x-x(M, q)\|_{\infty} \leq \frac{1+\|M\|_{\infty}}{c(M)}\|r(x)\|_{\infty}, \quad \text { for any } x \in R^{n}
$$

in $\|\cdot\|_{\infty}$. Moreover, we showed that the error bound (5.1) can be computed easily for some special matrix LCP. For instance, if $M$ is an H-matrix with positive diagonals, we have

$$
\mu(M):=\max _{d \in[0,1]^{n}}\left\|(I-D+D M)^{-1}\right\| \leq\left\|\tilde{M}^{-1} \max (\Lambda, I)\right\|
$$

where $\Lambda$ is the diagonal parts of $M$.

In this paper, we study the behavior of the solution $x(M, q)$ when there are some perturbations $\triangle M$ and $\triangle q$ in $M$ and $q$. In particular, we show

$$
\|x(M+\triangle M, q+\triangle q)-x(M, q)\| \leq \beta(M)\|\triangle M x(M+\triangle M, q+\triangle q)+\triangle q\| .
$$


The constants $\mu(M)$ and $\beta(M)$ play different roles, where the former is for computation of error bounds and the latter is for sensitivity analysis.

Theorem 2.2 proves that $\beta(M)$ is smaller than the Mathias-Pang constant $1 / c(M)$ for sensitivity and stability analysis [3]. Theorem 2.5 and Theorem 2.7 provide various interesting properties (1.5) -(1.7) of $\beta(M)$ when $M$ is an H-matrix with positive diangonals, M-matrix or symmetric positive definite matrix. These results show that the condition number $\kappa(M)$ is a measure of the sensitivity of the $\operatorname{LCP}(M, q)$. This means that if $\kappa(M)$ is small(large), then small changes in $M$ or $q$ result in small (large) changes in the solution $x(M, q)$ of the $\operatorname{LCP}(M, q)$.

When the $\operatorname{LCP}(M, q)$ is used in the modelling of a practical application, the matrix $M$ and vector $q$ often contain errors due to inaccurate data, uncertain factors, etc. Hence, to make $x(M, q)$ useful in practical, it is very important to obtain some sensitivity information of the solution. This is one reason why sensitivity analysis of the $\operatorname{LCP}(M, q)$ has been studied so extensively [3]. In the website http://www.st.hirosaki-u.ac.jp/ chen/ExamplesLCP.pdf, we provide numerical examples including free boundary problems [14] and traffic equilibrium problems $[3,6]$ to illustrate the practical value of the new pertubations bounds (1.1)-(1.7).

Acknowledgments. The authors are grateful to Prof. Z.Q. Luo and two anonymous referees for their helpful comments.

\section{REFERENCES}

[1] A. Berman and R. J.Plemmons, Nonnegative Matrices in the Mathematical Sciences, SIAM Publisher, Philadelphia, 1994.

[2] X. Chen And S. Xiang, Computation of error bounds for P-matrix linear complementarity problems, Math. Programming, 106(2006),pp. 513-525.

[3] R. W.Cottle, J. -S.Pang and R. E.Stone, The Linear Complementarity Problem, Academic Press, Boston, MA, 1992.

[4] J. E. Dennis, JR. And R. B. Schnabel, Numerical Methods for Unconstrained Optimization and Nonlinear Equations, SIAM Publisher, Philadelphia, 1996.

[5] F.FACChinei ANd J. -S.PAng, Finite-Dimensional Variational Inequalities and Complementarity Problems, I and II, Springer-Verlag, New York, 2003.

[6] M. C.FerRis AND J.S.PANG, Engineering and economic applications of complementarity problems, SIAM Rev., 39(1997),pp. 669-713.

[7] S. A.Gabriel AND J. J.Moré, Smoothing of mixed complementarity problems, Complementarity and Variational Problems: State of the Art, eds by M.C.Ferris and J.-S.Pang, SIAM Publications, Philadelphia, PA, 1997, pp.105-116.

[8] G. H. Golub and C. F. VAn Loan, Matrix Computations, Third Edition, The Johns Hopkins University Press, Baltimore, 1996.

[9] R. A.Horn and C. R.Johnson, Matrix Analysis, Cambridge University Press, 1985.

[10] R. A.Horn and C. R.Johnson, Topics in Matrix Analysis, Cambridge University Press, 1991.

[11] Z. Q. Luo, J. -S.PANG AND D. Ralph, Mathematical Programs with Equilibrium Constraints, Cambridge University Press, Cambridge,1996.

[12] R. Mathias And J. -S. PAng, Error bounds for the linear complementarity problem with a P-matrix, Linear Algebra Appl. 132(1990), pp. 123-136.

[13] L. QI, Convergence analysis of some algorithms for solving nonsmooth equations, Math. Oper. Res. 18(1993), pp. 227-244.

[14] U. SCHÄFER, An enclosure method for free boundary problems based on a linear complementarity problem with interval data, Numer. Func. Anal. Optim. 22(2001), pp. 991-1011.

[15] U. SCHÄFER, A linear complementarity problem with a P-matrix, SIAM Rev., 46(2004), pp. 189-201. 